\title{
CONTRIBUCIÓN AL DESARROLLO SOSTENIBLE LOCAL DE LOS PROYECTOS MDL EN EL SECTOR DE GENERACIÓN ELÉCTRICA POR BIOMASA: CASO INCAUCA S.A.*
}

\author{
CARLOS ANDRÉS VERGARA TAMAYO*** \& DIANA CAROLINA ORTIZ MOTTA*** \\ UNIVERSIDAD MILITAR NUEVA GRANADA
}

Recibido/ Received/ Recebido: 17/12/2014 - Aceptado/ Accepted / Aprovado: 24/11/2015

\section{Resumen}

La declaración presentada por la comunidad internacional a mediados del siglo XX frente al reconocimiento del fenómeno del cambio climático, dio origen a propuestas de la comunidad para la creación de mecanismos orientados al control y a la reducción de sus causas. Una de estas propuestas es el Protocolo de Kyoto, el cual tiene por objetivo la reducción de los Gases Efecto Invernadero (GEI) causantes del calentamiento global. Dicho protocolo planteó tres iniciativas, pero, tan solo una de ellas involucra la participación de países en vía de desarrollo como es el caso de Colombia; se trata de los Proyectos de Mecanismo de Desarrollo Limpio (MDL). En este sentido, el objetivo de este documento es comprender los aportes en términos del desarrollo sostenible local, a partir de la metodología multicriterio AHP, para el proyecto MDL de cogeneración y sustitución de combustible del Ingenio del Cauca S.A., registrado ante la Convención Marco de las Naciones Unidas para el Cambio Climático (CMNUCC.) De este trabajo se concluye que no existe una diferencia importante entre la situación "con proyecto" y la situación "sin proyecto", en otras palabras, la contribución al desarrollo social del Municipio de Miranda (Cauca), no está determinada por la puesta en marcha del proyecto MDL, sin embargo al analizar de manera global el ranking de las dos alternativas evaluadas es evidente que los actores involucrados prefieren la situación antes del proyecto que con este.

Palabras clave: Desarrollo sostenible local; MDL; Cambio climático; Protocolo de Kyoto; Análisis multicriterio.

\section{CONTRIBUTION TO LOCAL SUSTAINABLE DEVELOPMENT OF THE CDM PROJECTS IN THE BIOMASS POWER GENERA- TION SECTOR: INCAUCA S.A.CASE}

\begin{abstract}
The statement made by the international community in the mid-twentieth century against the recognition of the phenomenon of climate change, led to proposals from the community to create
\end{abstract}

Proyecto desarrollado en el Grupo de Estudios en Ciencias Económicas (CIE) de la Universidad Militar Nueva Granada (UMNG) Bogotá, Colombia. Resultado del proyecto de Investigación ECO-1216 denominado: "Proyectos del mecanismo de desarrollo limpio (MDL) en el sector de generación eléctrica por biomasa y su contribución al desarrollo sostenible en Colombia", aprobado y financiado por la Vicerrectoría de Investigaciones de la UMNG.

* Docente Investigador del Grupo CIE, Universidad Militar Nueva Granada (Bogotá-Colombia). Economista de la Universidad Nacional de Colombia (Medellín-Colombia), Magíster en Economía de Recursos Naturales y del Medio Ambiente de la Universidad de Concepción (Concepción-Chile). Dirección postal: Cra. 11 No. 101-80, Bogotá (Colombia), Centro de Investigaciones de la Facultad de Ciencias Económicas, Primer Piso, Bloque C. Teléfono: 6500000 Ext. 1306. Correo electrónico: carlos.vergara@unimilitar.edu.co

** Auxiliar de Investigación del Grupo CIE, Universidad Militar Nueva Granada, Economista de la Universidad Militar Nueva Granada (Bogotá-Colombia). Magíster en Estudios y Gestión del Desarrollo de la Universidad de la Salle (Bogotá-Colombia). Correo electrónico:diana.ortiz@unimilitar.edu.co 
mechanisms oriented to control and the reduce its causes. One of these proposals is the Kyoto Protocol, which aims at reducing greenhouse gas (GHG) emissions that cause global warming. This protocol outlined three initiatives, but only one of them involves the participation of developing countries as in the case of Colombia; they are the Clean Development Mechanism (CDM) Projects. In this sense, the objective of this document is to understand the contributions in terms of local sustainable development, from the multi-criteria methodology AHP for the cogeneration CDM project and fuel substitution of Ingenio del Cauca SA, registered with the United Nations Framework Convention on Climate Change (UNFCCC.) this paper concludes that there is no significant difference between the situation "with the project" and the situation "without the project", in other words, the contribution to the social development of the Municipality of Miranda (Cauca), is not determined by the implementation of the CDM project, however comprehensively analyzing the ranking of the two evaluated alternatives is evident that stakeholders prefer the situation before the project than with it.

Keywords: Local sustainable development; CDM; Climate change; Kyoto Protocol; Multicriteria analysis.

\title{
CONTRIBUIÇÃO AO DESENVOLVIMENTO SUSTENTÁVEL LOCAL DE PROJETOS MDL NO SETOR DE GERAÇÃO ELÉTRICA POR BIOMASA: CASO INCAUCA S.A.
}

\begin{abstract}
Resumo
A declaração apresentada pela comunidade internacional na metade do século XX frente ao reconhecimento do fenômeno da mudança climática deu origem a propostas de criação de mecanismos orientados ao controle e à redução de suas causas. Uma destas propostas é o Protocolo de Kyoto, que tem por objetivo a redução dos Gases Efeito Estufa causantes do aquecimento global. Este protocolo propôs três iniciativas, mas somente uma delas envolve a participação de países em via de desenvolvimento como é o caso da Colômbia. Trata-se dos Projetos de Mecanismo de Desenvolvimento Limpo (MDL). Neste sentido, o objetivo deste documento é compreender a contribuição em termos de desenvolvimento sustentável local, a partir da metodologia multicritério AHP, para o projeto MDL de cogeração e substituição de combustível do Talento do Cauca S.A., registrado ante a Convenção Marco das Nações Unidas para a Mudança Climática. Conclui-se neste trabalho que não existe uma diferença importante entre a situação "com projeto" e a situação "sem projeto". Em outras palavras, a contribuição ao desenvolvimento social do Município de Miranda (Cauca), não está determinada pela realização do projeto MDL. No entanto, ao analisar de maneira global o ranking das duas alternativas avaliadas, é evidente que os atores envolvidos preferem a situação anterior ao projeto do que com ele.
\end{abstract}

Palavras chave: Desenvolvimento sustentável local; MDL; Mudança climática; Protocolo de Kyoto; Análise multicritério.

Vergara, C. \& Ortiz, D. (2016). Contribución al desarrollo sostenible local de los proyectos MDL en el sector de generación eléctrica por biomasa: caso Incauca S.A. En: Revista de la Facultad de Ciencias Económica: Investigación y Reflexión. rev.fac.cienc.econ, XXIV (2), DOI: http://dx.doi. org/10.18359/rfce. 2218

JEL: P32, Q01, Q56, R11. 


\section{Introducción}

Las determinaciones internacionales frente al problema del calentamiento global y por consiguiente el cambio climático, permitieron el surgimiento de diversas alternativas de gestión ambiental, dentro de estas el Protocolo de Kyoto. Este surge bajo la Convención Marco de las Naciones Unidas Sobre Cambio Climático (CMNUCC) ${ }^{1}$, llevada a cabo en la ciudad de Kyoto (Japón) en $1997^{2}$, con el objetivo de reducir las emisiones de Gases Efecto Invernadero $(\mathrm{GEI})^{3}$ consideradas las causantes del problema del calentamiento global, en un 5\% durante un periodo de 2008 a 2012. El protocolo establece ciertos mecanismos que facilitan a los países industrializados alcanzar los compromisos adquiridos con acciones llevadas a cabo más allá de sus fronteras, dentro de éstos se encuentran los denominados proyectos de Mecanismo de Desarrollo Limpio (MDL) ${ }^{4}$, que a su vez, se convierten en el único mecanismo que permite que los países no industrializados hagan parte del protocolo y así alcanzar el objetivo de protección ambiental y de fomento y contribución del desarrollo sostenible (ONU, 1998).

Un planteamiento importante que surge a partir del Protocolo de Kyoto es la promoción del Desarrollo Sostenible, visto como una oportunidad para emplear energías con medios más limpios a los convencionales y así evitar la generación de emisiones de GEI. A partir de aquí y con el apoyo de nuevas propuestas sobre el Desarrollo Sostenible (ONU, 2012; SDSN, 2013 \& ONU, 2014), se pretenden revisar las cuatro dimensiones de éste: desarrollo económico, inclusión social, sostenibilidad ambiental y buena gobernabilidad, en un ejercicio de evaluación ex-post de los proyectos de Mecanismo de Desarrollo Lim-

1 Convención Marco de las Naciones Unidas para el Cambio Climático - CMNUCC. United Nations Framework Convention on Climate Change - UNFCCC.

2 A pesar de que el protocolo de Kyoto fue firmado el 11 de diciembre de 1997, solo entró en vigencia hasta que fue ratificado por el 55\% de las partes que el 16 de febrero de 2005 (De Quinto \& Ferreira, 2005).

3 Entre los Gases Efecto de Invernadero - GEI se encuentran: el dióxido de carbono (CO2), metano ( $\mathrm{CH} 4)$, óxido nitroso (N2O), hidrofluorocarbonos (HFC), perfluorocarbonos (PFC) y hexalfluoruro de azufre (SF6)

4 En inglés Clean Development Mechanism (CDM). pio (MDL) relacionados con energías limpias o renovables, dentro de las cuales se encuentra la energía generada a partir de biomasa, la cual se convierte en el objeto de análisis del presente documento.

Al respecto, Colombia cuenta con 6 proyectos MDL relacionados con energía generada a partir de biomasa y se ubica en el décimo tercer lugar a nivel mundial y el quinto a nivel de Latinoamérica y el Caribe (UNEP, 2014). En este sentido el Ingenio del Cauca S.A. (a partir de la cogeneración y sustitución de combustibles) se convierte en un caso de estudio interesante con características particulares lo que permite evidenciar la contribución de los proyectos de MDL en el sector de generación eléctrica por biomasa al desarrollo sostenible de Colombia. Dicha elección obedece entonces al análisis de la información contenida en el portal del PNUMA ${ }^{5}$ relacionado con la revisión de bases de datos $\mathrm{CDM} / \mathrm{JI}$ (UNEP RISOE CDM/JI Pipeline Analysis and Database) y los 95 Documentos de Diseño de Proyecto (PDD por sus siglas en inglés) ${ }^{6}$, presentados por Colombia ante la Convención Marco de las Naciones Unidas con corte a primero de Diciembre de 2014 y la información disponible de las empresas que tienen que ver con tales proyectos.

A pesar de que este documento reconoce que los postulados base para la creación tanto del Protocolo de Kyoto como de los Proyectos de Mecanismo de Desarrollo Limpio (MDL) que parten de doctos como Pigou o Coase, están soportados en la economía ambiental, no significa que la teoría que sustenta este documento sea ésta, por el contrario, y tal como se registra en el desarrollo metodológico, en la discusión, resultados y conclusiones de este escrito, es la Economía Ecológica área del conocimiento que reconoce un enfoque más holístico e interdisciplinar que busca imprimir la diferencia demostrando que el desarrollo económico no debe ser la única dimensión válida para el adelanto de iniciativas amigables con el ambiente de este tipo.

5 Programa de Naciones Unidas para el Medio Ambiente PNUMA, UNEP por sus siglas en inglés.

6 Un Documento de Diseño de Proyecto (PDD por sus siglas en inglés), es un formato que contiene toda la información del proyecto MDL y que es el único documento formal en la validación y registro del MDL ante las Naciones Unidas. 
Para el desarrollo de este documento se han dispuesto seis secciones, que le permitirán al lector comprender secuencialmente cómo los proyectos de Mecanismo de Desarrollo Limpio (MDL) en particular el proyecto "Cogeneración y Sustitución de Combustible en el Ingenio del Cauca S.A", contribuyen al desarrollo sostenible del municipio de Miranda (Departamento del Cauca). En tal sentido, el documento se compone entonces de una introducción, como bien se expone en esta primera sección. A manera seguida se presenta el marco teórico relacionado con la investigación, en el que además se encuentran las bases conceptuales; posteriormente se aborda la metodología denominada Análisis del Proceso Jerárquico (AHP, por su sigla en inglés). Continúa con la sección de resultados y el análisis de los hallazgos tanto cualitativos como cuantitativos y finaliza con una sección de conclusiones.

\section{Marco teórico}

\subsection{La problemática internacional del cambio climático}

A partir del reconocimiento del fenómeno del cambio climático por parte de la comunidad internacional se pronuncian los diferentes países a través de la Convención Marco de las Naciones Unidas sobre el Cambio Climático al definirlo como "un cambio de clima atribuido directa o indirectamente a la actividad humana que altera la composición de la atmósfera mundial y que se suma a la variabilidad natural del clima observada durante períodos de tiempo comparables" (ONU, 1992, p. 3).

Este proceso global se ha reconocido efectivamente como irreversible (Sánchez, citado en Canoura, 2009), esto debido a la acumulación de gases que generan el efecto invernadero y, por ende, aportan al cambio climático, en el momento ya existe una acumulación de emisiones con efectos irreversibles. En razón a lo anterior sus efectos y consecuencias no podrán ser contrarrestadas en su totalidad, tan sólo pueden ser atenuadas.

En Colombia, el Instituto de Meteorología y Estudios Ambientales -IDEAM-, como una de las instituciones gubernamentales determinantes, considera que: " $E l$ cambio climático es por lo tanto una de las amenazas más serias que atentan contra el bienestar y la salud humana, contra la economía y muchos de los ecosistemas naturales y, finalmente, contra el desarrollo humano sostenible" (IDEAM, 2002, p. 2). Esto representa claramente el efecto rebote ${ }^{7}$ de la degradación de los recursos naturales y el ambiente sobre el bienestar del ser humano.

Es importante mencionar que si bien existen algunas entidades encargadas de liderar estas actividades, todas las instituciones tanto nacionales como regionales deben fomentar la recolección, análisis y sistematización de la información del sector forestal, con el objetivo de revisar los mecanismos de flujo de información, hasta mejorar la calidad de los datos reportados; adicionalmente deben identificar los datos prioritarios que a nivel nacional, regional y local son útiles para el desarrollo de estudios nacionales e internacionales (IDEAM et al., 2006).

De acuerdo a lo anterior, el IDEAM en 2001, preparó la Primera Comunicación Nacional de Cambio Climático a presentar ante la Convención Marco de las Naciones sobre Cambio Climático-CMNUCC-, allí se presentaron resultados importantes que demuestran, que a pesar de que Colombia emite únicamente el 0,25\% de las emisiones globales será muy vulnerable a los efectos que se presenten por el Cambio Climático; el estudio prevé que para el año 2050 la temperatura promedio del aire aumentará entre 1 y $2^{\circ} \mathrm{C}$; las precipitaciones variarán aproximadamente un 15\%; desaparecerá el 78\% de la superficie de los nevados y el $56 \%$ de los páramos; y el mar ascenderá entre 40 y 60 centímetros (IDEAM et al., 2006)

Los avances y la buena disposición que Colombia ha demostrado frente al tema de cambio climático

\footnotetext{
El efecto rebote o paradoja de Jevons, fue mencionada por primera vez por William Stanley Jevons en su libro "The Coal Question" (La cuestión del carbón) en 1865, pretendiendo explicar el paradójico fenómeno de que al reducir el consumo de carbón de las máquinas de vapor gracias a mejoras tecnológicas, paradójicamente se aumentaba la demanda total. Estrictamente Alcott (2005, p. 8) la menciona como la paradoja que al "aumentar la eficiencia disminuye el consumo instantáneo pero incrementa el uso del modelo lo que provoca un incremento del consumo global".
} 
se pueden observar en la Segunda Comunicación Nacional ante la Convención Marco de las Naciones Unidas sobre Cambio Climático preparada por el Ministerio de Ambiente, Vivienda y Desarrollo Territorial, y el Instituto de Hidrología, Meteorología y Estudios Ambientales (IDEAM), allí queda la evidencia del reconocimiento que hemos tenido a nivel mundial, pues citan que: "En septiembre 18 de 2009, Colombia se convirtió en el quinto país de Latinoamérica y décimo segundo del mundo en proyectos elegibles bajo la modalidad MDL del Protocolo de Kyoto" (Barba et al., 2010, p. 184).

\subsection{Protocolo de Kyoto y Proyectos de Mecanismo de Desarrollo Limpio (MDL)}

Una de las iniciativas es el Protocolo de Kyoto, en las bases teóricas de este acuerdo se comprende la economía ambiental, fundamentalmente en los postulados de dos importantes economistas Arthur Cecil Pigou y Ronald H. Coase. El primero, desarrolló en 1920 su obra "Teoría del Bienestar" en la que incentiva al Estado a tomar medidas que mejoren la calidad de vida de sus ciudadanos, además reconocía que los mercados presentan externalidades tanto positivas como negativas, las cuales podrían mejorar mediante la creación de impuestos y subsidios. Este primer autor provee los asientos para penalizar a los países más contaminantes y al mismo tiempo premia a aquellos que mejoran sus prácticas en favor del ambiente (Alvater, 1998). Pero quizás quien más incidió en la determinación de esta política ambiental internacional fue el profesor Ronald $\mathrm{H}$. Coase, quien llevó a cabo un reconocido aporte en 1960, denominado: "El Problema del Costo Social", en el cual le resta protagonismo al Estado $e$ incentiva las condiciones de libre mercado a través de negociaciones descentralizadas, asignando al Estado un rol pasivo, esto es, de árbitro frente a los acuerdos voluntarios creados entre los generadores y receptores de contaminación.

Es en la Tercera Convención Marco de las Naciones Unidas sobre el Cambio Climático celebrada en 1997 donde 38 de los países más industrializados asumen compromisos de reducción de gases GEI hasta alcanzar un nivel promedio del $5,2 \%$ por debajo de los índices registrados para el año 1990, es- tos compromisos fueron recogidos en el "Protocolo de Kyoto" el cual fue adoptado en 1997 y ratificado en 2005. El protocolo de Kyoto se convirtió desde entonces en una de las principales muestras de compromiso y protección con el ambiente mediante objetivos que involucran un desarrollo sostenible ya que los gobiernos de los países firmantes actuaron bajo principios de equidad y responsabilidad común pero diferenciada aceptando restricciones frente a los niveles de emisiones de GEI y el compromiso de promover el desarrollo sostenible en otros países: subdesarrollados y en vías de desarrollo. A la fecha es el primer y único acuerdo vinculante que los países industrializados han ratificado y en donde se asumen reducciones cuantificadas de GEI como resultado del reconocimiento de su mayor responsabilidad en la crisis ambiental (ONU, 1998; Villavicencio, 2004).

Adicionalmente, investigaciones realizadas con anterioridad por organismos internacionales, determinaron que dentro de las principales causas que ocasionan el cambio climático se encuentra la acumulación de gases GEI en la atmósfera, por lo tanto en el protocolo se establece una serie de mecanismos para que los países industrializados puedan dar cumplimiento a los compromisos adquiridos. Para tal fin, el protocolo establece tres mecanismos de flexibilidad: el comercio de emisiones, la implementación conjunta (exclusivos para países industrializados), y el Mecanismo de Desarrollo Limpio (MDL). La alternativa de MDL, permite la posibilidad de que los países industrializados acompañen a los países en desarrollo con inversiones en proyectos que reduzcan GEI y promuevan el desarrollo sostenible en estos últimos, a la vez que el volumen de reducciones de GEI es reconocido para cumplir con los compromisos de los primeros (Villavicencio, 2004; Yamin, 2005; Stern, 2007; citados en Aleluia \& Leitão; 2009).

El objetivo de la implementación de proyectos MDL relaciona procesos de transferencia tecnológica para los países en vías de desarrollo, en especial en el campo de energías renovables y el manejo de desperdicios. Gran parte de los países que no han experimentado el MDL insisten en continuar con la tecnología que tradicionalmente utilizan, pero, aquellos que han tenido alguna experiencia con proyectos 
MDL logran combinarla con tecnología externa (Doranova, Costa \& Duysters, 2009). Por lo tanto la transferencia de tecnología se convierte en un objetivo indirecto de este tipo de proyectos, posibilitando el conocimiento y operación de tecnología local y de alguna manera, minimizando la importación y dependencia tecnológica en otros países.

\subsection{Los proyectos de Mecanismo de Desarrollo Limpio (MDL) y el desarrollo sostenible}

Si bien, el Mecanismo de Desarrollo Limpio (MDL) ha sido creado con el fin de atender dos objetivos simultáneamente: en primer lugar tratar de reducir las emisiones de Gases Efecto Invernadero (GEI) y en segundo lugar promover el desarrollo sostenible en los países no anexo I ${ }^{8}$ (Karakosta, Doukas \& Psarras 2009; Huang \& Barker, 2010; Sabogal, Hurtado \& Moreno, 2010), se hace necesario examinar la manera en que este cumple con su segundo objetivo bajo un enfoque holístico, por tanto a continuación se presentan algunas precisiones de cómo se alcanza el desarrollo sostenible a través de proyectos MDL.

Para Villavicencio (2004, p. 2), existen dos ideas fundamentales que predominan en el discurso sobre la contribución de los proyectos MDL al desarrollo sostenible de los países no anexo 1 o países en vía de desarrollo: "En primer lugar, los proyectos MDL, al reducir las emisiones de gases de efecto invernadero, promueven el desarrollo sustentable; y en segundo lugar los proyectos MDL, al atraer un flujo de inversión externa directa, son positivos para el logro de los objetivos de un desarrollo sustentable". De acuerdo a esto y al convertirse en canalizadores de inversión extranjera directa (IED), se asume que los efectos positivos se pueden desencadenar en: generación de divisas, transferencia de tecnología en beneficio del ambiente, efectos positivos en la balanza de pagos, manejo sostenible de los recursos, aumento de la inversión social, diversificación de la producción rural y apertura a nuevos mercados, generación de empleo e incluso disminución en la migración campesina (Mejía, 2005).

\footnotetext{
Países en vía de desarrollo.
}

El sin número de beneficios que surgen a partir de la inversión extranjera directa a causa de los proyectos MDL, se han justificado a través de la "Teoría de la filtración" propuesta por el Premio Nobel de Economía, Joseph Stiglitz en 2002, según la cual, los efectos positivos de la inversión extranjera directa, se filtran a través de toda la economía y llegan incluso a los estratos más pobres de la población (Villavicencio, 2004).

Sin embargo, Olsen \& Fenhann (2008) reconocen algunos de los inconvenientes presentados con los proyectos MDL en cuanto al desarrollo sostenible. $\mathrm{Se}$ ha comprobado que algunos proyectos MDL no logran los objetivos de desarrollo sostenible, para los que fueron creados, al estudiar las causas del incumplimiento es común encontrar desequilibrios entre los beneficios económicos que se obtienen en el mercado de carbono y los beneficios no económicos (sociales y ecológicos) que se pretenden con el logro del desarrollo sostenible. Otra de las causas referentes al no cumplimiento de los compromisos frente al desarrollo sostenible, surge en el momento en que van a ser verificadas las reducciones de GEI, este, es tal vez, uno de los momentos más importantes en el proceso, pues con la verificación del cumplimiento de reducciones se generan los $\mathrm{CERs}^{9}$ que posteriormente van a ser negociados en los mercados de carbono y de los cuales la empresa logra parte de su lucro económico; sin embargo, en el momento de la verificación no se exige ningún tipo de cumplimiento frente a los compromisos adquiridos en relación al desarrollo sostenible (Olsen \& Fenhann, 2008).

De esta manera se registran a la fecha de corte del presente trabajo (01 de diciembre de 2014), 8.647 proyectos MDL a nivel mundial, de los cuales el 30,28\% (2.618) manejan energía eólica, el 26,16\% (2.262) corresponden a hidroeléctricas y en el tercer lugar se encuentran los proyectos MDL, con una participación menor, que involucran energía

\footnotetext{
CER's son Certificados de Reducción de Emisiones que se miden como créditos equivalentes a una tonelada de $\mathrm{CO} 2$ los cuales son tenidos en cuenta para alcanzar los compromisos de reducción de emisiones adquiridos por los países en el Protocolo de Kyoto. Estos CER's son expedidos por las entidades operacionales designadas, bajo los parámetros y lineamientos de la Junta Directiva de MDL.
} 
por biomasa con un 9,03\% (781); de estos últimos Colombia cuenta con 6 proyectos y se ubica en el décimo tercer lugar a nivel mundial y el quinto a nivel de Latinoamérica y el Caribe, como se evidencia en la Tabla 1 (UNEP, 2014). Los proyectos registrados en Colombia se describen a continuación:

- MDL Retirado: Planta doble propósito de Propal: Evasión de metano y eficiencia energética.

- MDL Retirado: Cogeneración de azúcar, Ingenio Providencia.

- MDL Retirado: Cogeneración y desplazamiento de energía térmica por Incauca S.A.

- MDL Activo: Biomasa en la planta de cemento Caracolito por CEMEX Colombia.

- MDL Activo: Combustibles alternativos y proyectos de biomasa en la planta de cemento de Cúcuta por CEMEX Colombia.

- MDL Activo: Sustitución de combustible de carbón a residuos de bagazo de caña (biomasa) por Incauca S.A.
De acuerdo a lo anterior se evidencia que el proyecto MDL objeto de estudio en este documento "Sustitución de combustible de carbón a residuos de bagazo de caña (biomasa) por Incauca S.A.", se encuentra registrado, activo y con un tiempo de operación considerable, por lo tanto resulta oportuna y vigente la presente investigación.

Como se mencionó anteriormente, la presente investigación se apoya en el carácter multidimensional de desarrollo sostenible como lo describe Angulo (2010, p.4):

"Tal desarrollo será sostenible si vinculara las decisiones económicas con el bienestar social y ecológico, es decir, vincular la calidad de vida con la calidad del medio ambiente $y$, por lo tanto, con la racionalidad económica y el bienestar social. En otras palabras, el desarrollo es sostenible si mejora el nivel y la calidad de la vida humana al tiempo que garantiza y conserva los recursos naturales del planeta".

Tabla 1. Proyectos MDL con Biomasa por país

\begin{tabular}{|c|c|c|c|}
\hline \multicolumn{4}{|c|}{ Biomass CDM projects } \\
\hline No. & Country & Projects & MW \\
\hline 1 & India & 334 & 2584,893 \\
\hline 2 & China & 161 & 4331,01 \\
\hline 3 & Brazil & 54 & 1095,415 \\
\hline 4 & Malaysia & 43 & 276,25 \\
\hline 5 & Thailand & 31 & 469,862 \\
\hline 6 & Indonesia & 18 & 99,2 \\
\hline 7 & Vietnam & 16 & 36,5 \\
\hline 8 & Mexico & 14 & 165,2543 \\
\hline 9 & Chile & 13 & 234 \\
\hline 10 & Uruguay & 9 & 266,8 \\
\hline 11 & Argentina & 8 & 146,348 \\
\hline 12 & Pakistan & 7 & 27 \\
\hline 13 & Colombia & 6 & 26,2 \\
\hline 14 & Philippines & 5 & 52,5 \\
\hline 15 & Dominican Republic & 4 & 85,905 \\
\hline 16 & Honduras & 5 & 31,7 \\
\hline 17 & South Africa & 5 & 25,7 \\
\hline 18 & Sri Lanka & 5 & 25,5 \\
\hline 19 & Kenya & 4 & 43,125 \\
\hline 20 & Ecuador & 3 & 55,5 \\
\hline 21 & Costa Rica & 3 & 1,4 \\
\hline 22 & Morocco & 3 & o \\
\hline 23 & Panama & 2 & 0,5 \\
\hline 24 & Israel & 2 & 6,8 \\
\hline 25 & Egypt & 2 & 0 \\
\hline
\end{tabular}

Fuente: Tomado de CDM/JI.

\begin{tabular}{|r|l|r|r|}
\hline \multicolumn{4}{|c|}{ Blomass CDM projects } \\
\hline No. & \multicolumn{2}{|c|}{ Projectry } & MW \\
\hline 26 & Singapore & 2 & 2,81 \\
\hline 27 & Uganda & 2 & 0 \\
\hline 28 & El Salvador & 2 & 78,5 \\
\hline 29 & Peru & 2 & 37,5 \\
\hline 30 & Senegal & 2 & 25 \\
\hline 31 & Côte d'lvoire & 2 & 46 \\
\hline 32 & Swaziland & 1 & 20 \\
\hline 33 & Cambodia & 1 & 1,5 \\
\hline 34 & Nicaragua & 1 & 55 \\
\hline 35 & Bangladesh & 1 & 0 \\
\hline 36 & Guyana & 1 & 10 \\
\hline 37 & Guatamala & 1 & 0 \\
\hline 38 & Macedonia & 0 & 2,5 \\
\hline 39 & Moldova & 1 & 0 \\
\hline 40 & South Korea & 1 & 3 \\
\hline 41 & Tanzania & 1 & 15 \\
\hline 42 & Serbia & 0 & 7,5 \\
\hline 43 & Nigeria & 1 & 0 \\
\hline 44 & Sierra leone & 1 & 32 \\
\hline 45 & Tunesia & 1 & 0 \\
\hline 46 & Paraguay & 0 & 0 \\
\hline 47 & Lao PDR & 0 & 0 \\
\hline 48 & Mongolia & 0 & 0 \\
\hline & Total & 781 & 10423,67 \\
\hline & & & \\
\hline
\end{tabular}


Así mismo, dentro de las iniciativas para promover el desarrollo, el Secretario General de Naciones Unidas, Ban Ki-moon, creó la Red de Soluciones para el Desarrollo Sostenible (SDSN, 2013), con el objeto de movilizar el conocimiento global científico y tecnológico en los retos de desarrollo sostenible, incluidos el diseño y la implementación de la agenda global para el desarrollo sostenible post-2015. En dicha agenda la SDSN reconoce la visión Río+20 como un concepto integral que aborda cuatro dimensiones: sostenibilidad ambiental, inclusión social, desarrollo económico, y buena gobernabilidad.

De esta manera, Colombia también le apuesta a la redefinición de los Objetivos del Desarrollo del Milenio por Objetivos de Desarrollo Sostenible, iniciativa de la ONU donde se otorga suficiente importancia al componente ambiental dentro de las políticas internacionales como tal. Es así como en el país surge la Comisión interinstitucional de alto nivel para el alistamiento y efectiva implementación de los objetivos de desarrollo sostenible que pretende canalizar el apoyo de la cooperación internacional e incrementar el beneficio de la misma en función del desarrollo y las prioridades del país (ONU, 2012; SDSN, 2013; DNP, 2015).

Frente a esto, es importante tener en cuenta los factores clave que permiten lograr una gestión exitosa frente al desarrollo sostenible en las economías emergentes, en tal sentido (Nuschler, 2005, citado en Cotte \& Pardo, 2011), menciona algunos como la modernización de la producción, el apalancamiento de capital y la transferencia de conocimientos técnicos; asimismo aclara que, la gestión adecuada de estos factores debería generar una transformación radical de la economía.

Lo que debe destacarse de cualquier posición frente al desarrollo sostenible, es el llamado a cambiar las estrategias que se han venido desarrollando hasta ahora, desde los diferentes aportes disciplinares; se hace necesario entonces actuar frente a este concepto cambiante a lo largo del tiempo que garantice la estabilidad social y ecológica de las diferentes generaciones. En este sentido, las nuevas actuaciones deberán estar focalizadas principalmente hacia los países industrializados pues son los mayores consumidores de recursos naturales y los que más des- perdicios generan (Pierri, 2001; Angulo, 2010). El primer paso en esta línea debería darse en aras de evidenciar la responsabilidad de los países desarrollados y no desarrollados pero así también en verificar los resultados e incluso reevaluar las estrategias de orden internacional y su impacto regional y local, como corresponde al presente caso de estudio.

Finalmente, este acápite ofrece las bases para conceptualizar el problema global del cambio climático destacando tan solo una de las herramientas que se han empleado en la mitigación de este: El Protocolo de Kyoto. Los proyectos de Mecanismo de Desarrollo Limpio (MDL), dirigidos hacia los países en vías de desarrollo como Colombia pretenden no solo disminuir las emisiones de GEI sino también promover el desarrollo sostenible. En este sentido se justifica la necesidad de comprender los aportes en términos del desarrollo sostenible local, desde un enfoque alternativo denominado Ciencia Post-normal (el cual se ampliará más adelante) y la economía ecológica a partir de la metodología de análisis multicriterial, método AHP, como se aclara en la siguiente sección, para el proyecto MDL de cogeneración y sustitución de combustible del Ingenio del Cauca S.A., registrado ante la Convención Marco de las Naciones Unidas para el Cambio Climático (CMNUCC).

\section{Metodología}

Las décadas de los setentas y ochentas son consideradas el punto oficial de partida de las metodologías más completas para estudiar los problemas económicos, sociales y ambientales que requieren la valoración en más de un escenario. Una de estas metodologías es denominada Análisis Comparativo Cualitativo (QCA), citada por primera vez por Charles Ragin en 1987, esta metodología reúne un conjunto de técnicas lógicas para realizar análisis cualitativos de causalidad (Ragin, 1987, 2008, citados en Cotte \& Pardo, 2011).

Pese a esto, Funtowicz \& Ravetz (2004), en sus investigaciones sobre el problema de la sustentabilidad, y reconociendo las múltiples fuentes de conocimiento existentes, logran construir un nuevo marco analítico el cual es llamado "Ciencia Post-normal" que orienta la integración del conocimiento de sentido común en 
el procesamiento de información requerido para la comprensión de los fenómenos socioambientales. Y es bajo este enfoque que se pueden realizar estudios en el campo social facilitando las distintas perspectivas de los actores sociales; como por ejemplo, los modelos de análisis multicriterio.

Es así, como el análisis multicriterio, busca integrar las diferentes dimensiones de la realidad de una problemática, con el fin de dar una visión integral y un mejor acercamiento a la realidad. Esta metodología es adecuada para la toma de decisiones frente a "conflictos sociales, económicos y objetivos de conservación del medio ambiente, y además cuando confluyen una pluralidad de escalas de medición (físicas, monetarias, cualitativas, etc.)" (Saaty, 1977; Falconí \& Burbano, 2004).

Debido a que esta metodología requiere la agregación de opiniones de los diferentes actores de una problemática, surge un enfrentamiento entre lo monocriterial y lo multicriterial. Sin embargo, esta última se caracteriza porque el decisor tiene mayor libertad para expresar sus ideas, las opiniones se hacen más realistas y menos rígidas, de esta manera, al final el análisis multicriterio termina imponiéndose, aun cuando no todos los actores muestren la misma sensibilidad frente a los diferentes criterios (Saaty, 1987; Fernández \& Escribano, 2011), esto permite finalmente capturar una interesante diversidad de posturas de acuerdo a los aspectos a evaluar.

De otro lado, algunos organismos regionales han aportado a la literatura contemporánea mencionando la aplicación de estas metodologías como se observa en Pacheco \& Contreras (2008, p. 14) en su documento Manual Metodológico de Evaluación Multicriterio para Programas y Proyectos avalado por la serie de manuales CEPAL, los cuales reafirman que: "las técnicas de Evaluación Multicriterio permiten trabajar con varios criterios a la vez, identificar la importancia relativa de cada uno y evaluar entre distintas alternativas de proyecto, independiente de la naturaleza de los factores que están implícitos en una situación específica".

Para Comas et al. (2011, p. 3), la aplicación de los métodos multicriterio han pasado de ser una herra- mienta encargada de tomar la decisión (MCDM Multi Criteria Decision Making), a una herramienta de apoyo a la toma de decisiones (MCDA Multi Criteria Decision Aid) (Pacheco \& Contreras 2008; Saaty, 2003).

En tal sentido, Fernández \& Escribano (2011), analizan los dos modelos de decisión de análisis multicriterio, a fin de presentar sus contribuciones específicas en la investigación operativa; por un lado está "La toma de decisiones multicriterio (MCDM)", cuyo objetivo es describir o descubrir algo que pueda ser considerado como una entidad fija y siempre presente, ayudando al decisor a tomar una decisión correcta (siempre que la racionalidad de los axiomas sea aceptada por el decisor) y por otro lado "La ayuda a la decisión multicriterio (MCDA)", su objetivo es construir o crear algo que no pre-existe, debe ser fiable para ayudar a un actor en la toma de decisiones, iluminando el comportamiento del decisor ofreciéndole argumentos capaces de reforzar o debilitar sus propias convicciones.

La decisión multicriterio es apropiada en situaciones en las que un decisor debe realizar elecciones en presencia de múltiples criterios y múltiples actores, para una mayor comprensión, el decisor (sea individual o colectivo), debe elegir entre varias posibilidades que son denominadas "alternativas", el conjunto de alternativas se denomina "conjunto de elección" y en este conjunto existen diferentes puntos de vista llamados "criterios", muchos de ellos contradictorios; es así cómo se constituye un sistema multicriterio en donde finalmente el decisor al aceptar un punto de vista no podrá escoger la misma alternativa en presencia de otro criterio (Fernández \& Escribano, 2011).

Pacheco \& Contreras (2008), plantean distintos métodos de agregación que modelizan las preferencias a través de una función valor dentro de los cuales se encuentra el proceso analítico jerárquico o método AHP (Analytic Hierarchy Process) ${ }^{10}$, que conduce al sistema a un objetivo deseado como la solución de conflictos, un desempeño eficiente o

10 Conocido también como método mixto, por la posibilidad de relacionar información tanto cuantitativa como cualitativa. 
simplemente la felicidad total; este método posee tres principios fundamentales que dirigen el proceso de evaluación: i) construcción de jerarquías; ii) establecimiento de prioridades; y iii) consistencia lógica. En este sentido la metodología AHP se convierte en una herramienta determinante a la hora de evaluar las contribuciones correspondientes del proyecto MDL del Ingenio del Cauca S.A sobre el desarrollo sostenible local en el área de estudio previamente especificada.

\subsection{Desde lo cualitativo: instrumentos de reco- lección de información}

La metodología de análisis multicriterio llevada a cabo, permitió involucrar los actores relacionados con el proyecto MDL que tienen que ver con la generación eléctrica por biomasa, para poder establecer una aproximación a la contribución de estos al desarrollo sostenible. Dichos actores fueron seleccionados de acuerdo a un "Muestreo por Conveniencia", esta técnica de muestreo no probabilístico consiste en seleccionarlos conforme a lo establecido por el investigador según su accesibilidad o proximidad, no por que hayan sido seleccionados mediante un criterio estadístico (Casal \& Mateu, 2003).

Dentro de las razones por las cuales se toma la decisión de emplear dicha técnica se consideraron los paradigmas metodológicos de investigación mencionados, así como los límites de tiempo y de presupuesto, de tal manera que los diferentes tipos de actores (mencionados a continuación) participaran y se reflejarán en el ejercicio de investigación: representante de la compañía Incauca S.A., representante de la Alcaldía de Miranda (Cauca), comunidad de la vereda El Ortigal, y comunidad de la vereda Santa Ana.

En este sentido se diseñaron y aplicaron los diferentes instrumentos cuantitativos y cualitativos como: encuestas, entrevistas, grupos focales y análisis de información secundaria ${ }^{11}$. En todos los casos se manejaron dos escenarios: un escenario sin proyecto MDL, es decir, donde se contemple la situación

11 El diseño de estos instrumentos puede observarse en el Anexo. antes implementar o desarrollar el proyecto y un segundo escenario con proyecto MDL, después de la implementación del mismo.

Es de anotar que la definición de escalas de calificación se basó en la escala fundamental de Saaty (1987), en la cual se plantea que existe un límite psicológico según el cual las personas acostumbran a realizar juicios de valor en una escala de 1 a 9 (aproximadamente) siguiendo una escala lineal; es importante aclarar que esta tabla se utiliza para la comparación par a par, para cada uno de los criterios y entre cada una de las alternativas de selección.

Las entrevistas relacionadas se realizaron a dos de los principales actores: Incauca S.A. y la Alcaldía del Municipio de Miranda. De otro lado las encuestas se aplicaron a todos los actores. Esta fue dividida en tres (3) partes así: La primera parte está compuesta por diez (10) preguntas en las que se debe calificar de 1 a 5 , siendo 1 la calificación más baja y 5 la calificación más alta. La segunda parte está compuesta por siete (7) preguntas, las cuales contienen diferentes opciones de respuesta como: expresiones en porcentaje, cantidades y otros. Y la tercera y última parte, busca que se ordenen algunos criterios (ranking) de acuerdo a su importancia de 1 a 7 , siendo 1 la calificación menos importante y 7 la calificación más importante.

\subsection{Unificación de información cualitativa y cuantitativa}

En la última parte del tratamiento de la información fue necesario homogeneizar cuantitativa y cualitativamente las diferentes fuentes de información, esto obedeció al proceso de triangulación de fuentes que permitiera confrontar las diferentes fuentes de información obtenida y luego re-escalarlas de 1 a 5 (Sandoval, 2002).

\subsubsection{Información secundaria}

En cuanto al análisis del informe de sostenibilidad de Incauca S.A., los resultados que la empresa muestra en este tienen que ver con la presencia del proyecto MDL, la información no se encuentra completa para los 17 indicadores evaluados, y por esta razón 
se presenta un vacío, debido también a que no se cuenta con informes de sostenibilidad que se hayan presentado antes de implementarse el proyecto MDL para poder realizar una comparación entre las alternativas $\sin y$ con proyecto.

Para la empresa Incauca S.A., el proyecto MDL es muy favorable principalmente en cuanto a la disponibilidad de energía eléctrica que al ser generada con biomasa contribuye con el mejoramiento de la calidad de vida de los colombianos. A través de un uso racional y eficiente de la energía, se logra una disminución en la emisión de gases nocivos para la salud; adicionalmente se cuenta con una iniciativa planteada por la empresa y acogida por la comunidad de Padilla (vereda ubicada a una hora de la planta), la cual consiste en el desarrollo y puesta en marcha de una cooperativa de corte y transporte de hoja de caña (residuos utilizados en el nuevo proceso de sustitución de combustibles) (Incauca S.A., 2011).

En materia de energía, Incauca S.A., es auto-sostenible. El bagazo de la caña resultante de la molienda se emplea en las calderas para ser utilizado como combustible en la generación del vapor de alta presión, que se emplea en las turbinas de los molinos para lograr su movimiento y en los turbogeneradores para generar la energía eléctrica que requiere la Empresa. El excedente se vende a la red pública. El bagazo sobrante en esta etapa, es empleado en la industria papelera. El vapor de escape de las turbinas se emplea en las operaciones de evaporación y cocimiento de los jugos azucarados. Los residuos orgánicos generados en la producción de azúcar y etanol como cachaza, ceniza, basuras de patios de caña y vinaza concentrada, se transforman en abono orgánico (compost) aplicable en la agricultura como mejorador del suelo (Incauca S.A., 2011).

Teniendo en cuenta el informe de sostenibilidad (Información secundaria) de la empresa Incauca S.A., la alternativa con proyecto MDL resulta más favorable. Debido a que no se contó con informes de sostenibilidad de Incauca S.A., antes de implementarse el proyecto MDL, se tomó como referencia para evaluar la alternativa sin proyecto, la entrevista rea- lizada al Ingeniero John Jairo León, persona encargada de implementar y monitorear el proyecto MDL en Incauca S.A., por lo que se concluyó que la alternativa sin proyecto es menos favorable para Incauca S.A.

\subsubsection{Entrevistas y grupos focales}

Se llevó a cabo la dinámica del grupo focal con las comunidades de las veredas Santa Ana y El Ortigal, ubicadas en el Municipio de Miranda, debido a que estas veredas son las más cercanas al ingenio, tan solo hasta en la etapa de campo se evidencio que dichas veredas decidieron no hacer parte del proyecto, pues desde que se socializo el mismo en 2008 , la comunidad no encontró suficiente motivación en el componente social que el proyecto proponía. Lo que se pudo observar, teniendo en cuenta la percepción por parte de la comunidad, es que la alternativa sin proyecto MDL es más favorable, debido a que la comunidad que se vio beneficiada con el proyecto MDL se determinó fuera del área de estudio.

En cuanto a la Alcaldía de Miranda, se reconocen aportes del proyecto MDL, se afirma que es favorable y ha beneficiado de manera importante a la comunidad aledaña, argumenta además que hay un problema de acuerdo a las dinámicas sociales y económicas, donde las comunidades siempre exponen algunas actitudes renuentes a las acciones de la empresa, lo cual genera choques importantes dada la misma concepción del desarrollo local.

Los grupos focales tienen como objetivo conocer la mayor cantidad de información tradicional tanto de la actividad cotidiana de los pobladores como de lo desarrollado por la empresa en esa zona. Finalmente, los grupos focales se desarrollaron con los recolectores de caña de azúcar de las dos veredas más cercanas al área de influencia donde se lleva a cabo el proyecto: El Ortigal y Santa Ana. No se logró realizar el grupo focal con la comunidad de Padilla, pues esta no aparecía registrada en el PDD, y en el momento de la visita en campo se presentaron serios problemas de orden público en la zona. 


\subsection{Análisis multicriterio como herramienta de evaluación del proyecto MDL Incauca S.A.}

Al usar la evaluación multicriterio (Multiple Criteria Decision Aid o MCDA) para el caso de estudio, específicamente aplicando el método de proceso de jerarquía analítica (Analytic Hierarchy Process o AHP), apoyado en el manual de la CEPAL de Pacheco \& Contreras (2008), con algunas modificaciones que se presentan en la Ilustración 1.

De esta manera se analizaron e incluyeron las cuatro dimensiones del desarrollo sostenible, planteadas en la última cumbre de Río + 20 que culminó en junio de 2012 (Dimensión de Sostenibilidad Ambiental, Inclusión Social, Desarrollo Económico y Gobernabilidad), a las cuales les fueron asignados siete (7) criterios relevantes para evaluar la contribución del MDL Incauca S.A. al desarrollo sostenible del Municipio de Miranda: i) efectos en la gestión ambiental percibidos por el uso de los residuos de cosecha; ii) generación de beneficios no monetarios a partir de los residuos de cosecha; iii) inversión atraída para el aprovechamiento de residuos de cosecha; iv) generación y calidad de empleo en actividades relaciona- das con el uso de residuos de cosecha; v) beneficios netos generados por los residuos de cosecha; vi) incentivos para la adopción de tecnologías y procesos técnicos en el aprovechamiento de residuos de cosecha; vii) fortalecimiento de organizaciones civiles $y$ de procesos participativos para el uso de residuos de cosecha; de igual manera se establecieron los indicadores correspondientes a cada uno de estos criterios de forma jerárquica tal como se muestra en la Tabla 2.

Desde la postura positivista la decisión de aplicar el muestreo por conveniencia como técnica no probabilística relaciona un sesgo importante debido a que la muestra consultada no sería suficientemente representativa de acuerdo a la población estudiada y asimismo comprometería la aleatoriedad en la selección correspondiente, sin embargo desde la ciencia posnormal, en la cual se enmarca el presente ejercicio de investigación, la subjetividad presentada durante el proceso de implementación correspondiente es inherente a la metodología empleada, como sucede con cualquier ejercicio de investigación, lo cual no compromete la validez de los resultados y hallazgos relacionados. Pese a estas

Ilustración 1. Metodología de análisis multicriterio aplicada al proyecto MDL de generación de energía eléctrica, a partir de los residuos de caña de azúcar por parte de Incauca S.A.

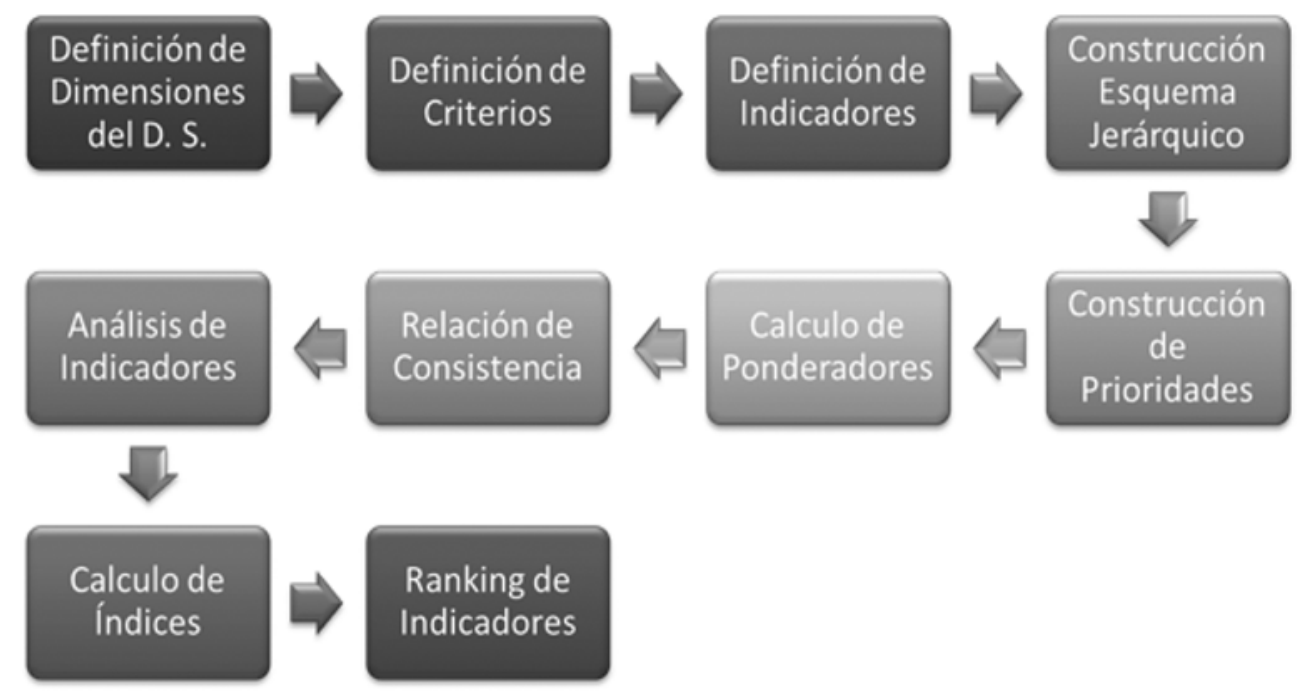

Fuente: Adaptación a partir de Pacheco \& Contreras (2008). 
Tabla 2. Conformación de dimensiones, criterios e indicadores

\begin{tabular}{|c|c|c|}
\hline $\begin{array}{l}\text { Dimensiones del Desarrollo } \\
\text { Sostenible }\end{array}$ & Criterio & Indicador \\
\hline \multirow{4}{*}{ Sostenibilidad Ambiental } & \multirow{4}{*}{$\begin{array}{l}\text { Efectos en la gestión ambiental percibidos por el } \\
\text { uso de los residuos de cosecha. }\end{array}$} & Percepción de los efectos en la gestión del agua. \\
\hline & & Percepción de los efectos en la gestión del suelo. \\
\hline & & Percepción de los efectos en la gestión del aire. \\
\hline & & Percepción de los efectos en la gestión de la biodiversidad. \\
\hline \multirow{2}{*}{ Inclusión Social } & \multirow{2}{*}{$\begin{array}{l}\text { Generación de beneficios no monetarios a partir de } \\
\text { los residuos de cosecha. }\end{array}$} & $\begin{array}{l}\text { Número de programas de inclusión social asociados al uso de } \\
\text { residuos de cosecha. }\end{array}$ \\
\hline & & $\begin{array}{l}\text { Efectividad de los programas de inclusión social asociados al } \\
\text { uso de residuos de cosecha. }\end{array}$ \\
\hline \multirow{8}{*}{ Desarrollo Económico } & $\begin{array}{l}\text { Inversión atraída para el aprovechamiento de Bio- } \\
\text { masa. }\end{array}$ & $\begin{array}{l}\text { Capitales atraídos para el aprovechamiento de residuos de } \\
\text { cosechas. }\end{array}$ \\
\hline & \multirow{3}{*}{$\begin{array}{l}\text { Generación y calidad de empleo en actividades rela- } \\
\text { cionadas con el uso de residuos de cosecha. }\end{array}$} & $\begin{array}{l}\text { Empleos generados por el manejo dado a los residuos de co- } \\
\text { sechas. }\end{array}$ \\
\hline & & $\begin{array}{l}\text { Duración de contratos del empleo generado por el manejo } \\
\text { dado a los residuos de cosechas. }\end{array}$ \\
\hline & & $\begin{array}{l}\text { Prestaciones sociales y seguridad social del empleo generado } \\
\text { por el manejo dado a los residuos de cosechas. }\end{array}$ \\
\hline & \multirow{2}{*}{$\begin{array}{l}\text { Beneficios netos generados por los residuos de } \\
\text { cosecha. }\end{array}$} & $\begin{array}{l}\text { Costo-beneficio (económico) en uso de residuos de cose- } \\
\text { chas. }\end{array}$ \\
\hline & & $\begin{array}{l}\text { Nivel de satisfacción por los beneficios económicos genera- } \\
\text { dos a partir de los residuos de cosecha. }\end{array}$ \\
\hline & \multirow{2}{*}{$\begin{array}{l}\text { Incentivos para la adopción de tecnologías y pro- } \\
\text { cesos técnicos en el aprovechamiento de residuos } \\
\text { de cosecha. }\end{array}$} & $\begin{array}{l}\text { Número de programas de apoyo para la adopción de tecnolo- } \\
\text { gías y procesos técnicos en el uso de residuos de cosecha. }\end{array}$ \\
\hline & & $\begin{array}{l}\text { Efectividad de los programas para la adopción de tecnologías } \\
\text { y procesos técnicos en el uso de residuos de cosecha. }\end{array}$ \\
\hline \multirow{3}{*}{ Gobernabilidad } & \multirow{3}{*}{$\begin{array}{l}\text { Fortalecimiento de organizaciones civiles y de pro- } \\
\text { cesos participativos para el uso de residuos de } \\
\text { cosecha. }\end{array}$} & $\begin{array}{l}\text { Número de procesos participativos implementados en la ges- } \\
\text { tión ambiental de residuos de cosecha. }\end{array}$ \\
\hline & & $\begin{array}{l}\text { Número de pobladores organizados o asociados o que parti- } \\
\text { cipan de procesos de planificación del manejo o aprovecha- } \\
\text { miento de los residuos de cosecha. }\end{array}$ \\
\hline & & $\begin{array}{l}\text { Efectividad de iniciativas que fomentan la participación de po- } \\
\text { bladores en el manejo de residuos de cosecha. }\end{array}$ \\
\hline
\end{tabular}

Fuente: Elaboración propia. 
necesarias discusiones metodológicas, se aplica la metodología AHP de manera rigurosa haciendo uso de esta técnica de selección muestral, reconociendo de antemano algunas potencialidades y limitaciones como bien lo resumen Vergara \& Lozada (2015) en la Tabla 3.

\section{Resultados y análisis}

\subsection{Resultados trabajo de campo}

En la primera parte del instrumento Encuesta, se puede observar la calificación generada por cada actor para cada indicador evaluado con dos escenarios posibles (sin proyecto MDL y con proyecto MDL) y con un rango de calificación de 1 a 5 , siendo el 1 el menos importante y el 5 el más importante:

- Los indicadores de sostenibilidad de un escenario sin proyecto son más favorables pues se afectan menos los recursos naturales de la zona y hay más opciones de empleo.

- Los indicadores sociales, en los dos escenarios (con y sin proyecto MDL), tienen muy bajas calificaciones, es decir que así exista o no exista el proyecto se afectan los recursos naturales sin implicaciones importantes sobre el empleo. A excepción de los beneficios económicos correspondientes a la dimensión de desarrollo económico y los indicadores ambientales correspondientes a la dimensión de sostenibilidad ambiental.

Los indicadores de gobernabilidad en los dos escenarios presentan una calificación muy baja, es decir con proyecto MDL o en ausencia de este el resultado para los indicadores no es favorable.

Dentro del procedimiento metodológico, posterior al trabajo de campo, se define la construcción de prioridades a través del ranking de los actores los cuales ordenaron los criterios de acuerdo a su nivel de importancia, este método es conocido como estandarización sencilla, a partir de allí se construyó la matriz en donde las columnas $(\mathrm{C} 1, \ldots \mathrm{C} 7)$ represen$\tan$ los criterios y las filas las encuestas $(\mathrm{E} 1, \ldots \mathrm{E} 18)$ aplicadas (Tabla 4).

Tabla 3. Potencialidades y limitaciones de la metodología

\begin{tabular}{|l|l|}
\hline $\begin{array}{c}\text { Potencialidades de la Metodología de Análisis Multicriterio por el } \\
\text { método de Proceso de Jerarquía Analítica (AHP) }\end{array}$ & $\begin{array}{l}\text { Limitaciones de la Metodología de Análisis Multicriterio por el método } \\
\text { de Proceso de Jerarquía Analítica (AHP) }\end{array}$ \\
\hline Pluralidad de Percepciones & Subjetividad \\
\hline Captura de Preferencias & Generalidad \\
\hline Procedimiento Riguroso & Limitaciones Cognitivas \\
\hline Toma de Decisiones & Sesgo en la Selección de Actores \\
\hline Flexibilización & Asimetría \\
\hline Convergencias y Divergencias & Presupuesto \\
\hline
\end{tabular}

Fuente: Elaboración propia a partir de Vergara \& Lozada (2015). 
Tabla 4. Construcción de prioridades

\begin{tabular}{|l|c|c|c|c|c|c|c|}
\hline & c1 & c2 & c3 & c4 & c5 & c6 & c7 \\
\hline E1 & 7 & 3 & 6 & 1 & 2 & 4 & 5 \\
\hline E2 & 5 & 4 & 3 & 7 & 6 & 1 & 2 \\
\hline E3 & 5 & 3 & 6 & 7 & 2 & 4 & 1 \\
\hline E4 & 7 & 3 & 6 & 1 & 2 & 4 & 5 \\
\hline E5 & 5 & 3 & 6 & 7 & 2 & 4 & 1 \\
\hline E6 & 5 & 3 & 6 & 7 & 2 & 4 & 1 \\
\hline E7 & 4 & 5 & 3 & 7 & 6 & 1 & 2 \\
\hline E8 & 4 & 5 & 3 & 7 & 6 & 1 & 2 \\
\hline E9 & 4 & 5 & 3 & 7 & 6 & 1 & 2 \\
\hline E10 & 1 & 2 & 7 & 6 & 5 & 4 & 3 \\
\hline E11 & 3 & 5 & 4 & 7 & 6 & 2 & 1 \\
\hline E12 & 3 & 5 & 4 & 7 & 6 & 2 & 1 \\
\hline E13 & 3 & 1 & 5 & 7 & 2 & 6 & 4 \\
\hline E14 & 2 & 1 & 6 & 7 & 3 & 5 & 4 \\
\hline E15 & 2 & 5 & 1 & 7 & 6 & 3 & 4 \\
\hline E16 & 5 & 4 & 3 & 7 & 2 & 1 & 6 \\
\hline E17 & 3 & 4 & 2 & 7 & 1 & 6 & 5 \\
\hline E18 & 3 & 4 & 2 & 7 & 1 & 6 & 5 \\
\hline PR0MED10 & 4 & 4 & 4 & 6 & 4 & 3 & 3 \\
\hline
\end{tabular}

\begin{tabular}{|c|c|}
\hline CRITERIOS & CALIFICACIÓN \\
\hline 1 & menos importante \\
\hline 2 & \\
\hline 3 & \\
\hline 4 & \\
\hline 5 & \\
\hline 6 & \\
\hline 7 & más importante \\
\hline
\end{tabular}

\begin{tabular}{|l|}
\hline C: Criterios \\
\hline E: Encuestas \\
\hline
\end{tabular}

Fuente: Elaboración propia.

\subsection{Resultados tratamiento de datos}

De otro lado, el cálculo de ponderadores se lleva a cabo transformado la matriz anterior en ceros (0) y unos (1) de acuerdo a la relación de importancia entre las columnas y las filas, resaltando la relación positiva con unos y relación negativa con ceros, tal como se muestra en la Tabla 4. Para el cálculo de los ponderadores simplemente se suman las filas y columnas y se calcula el porcentaje que cada uno representa en el total.
Para llevar a cabo este análisis se construyó una matriz de comparaciones, donde se ingresaron los juicios respecto a la importancia relativa de los criterios. Debido a que fue posible determinar qué criterio era más importante que otro, se utilizó el método sencillo de estandarización, para completar la matriz de comparaciones con ceros y unos según correspondiera, según lo muestra la Tabla 5 (Pacheco \& Contreras, 2008, p. 63). 
Tabla 5. Método sencillo de estandarización

\begin{tabular}{|c|c|c|c|c|c|c|c|c|c|}
\hline & C1 & C2 & C3 & C4 & C5 & C6 & C7 & SUMA & PONDERADOR \\
\hline $\mathrm{C} 1$ & & 0 & 0 & 1 & 0 & 0 & 0 & 1 & 0.07 \\
\hline C2 & 0 & & 0 & 1 & 0 & 0 & 0 & 1 & 0.07 \\
\hline C3 & 0 & 0 & & 1 & 0 & 0 & 0 & 1 & 0.07 \\
\hline C4 & 0 & 0 & 0 & & 0 & 0 & 0 & 0 & 0.00 \\
\hline C5 & 0 & 0 & 0 & 1 & & 0 & 0 & 1 & 0.07 \\
\hline C6 & 1 & 1 & 1 & 1 & 1 & & 0 & 5 & 0.36 \\
\hline $\mathrm{C7}$ & 1 & 1 & 1 & 1 & 1 & 0 & & 5 & 0.36 \\
\hline SUMA & 2 & 2 & 2 & 6 & 2 & 0 & 0 & & \\
\hline PONDERADOR & 0.14 & 0.14 & 0.14 & 0.43 & 0.14 & 0.00 & 0.00 & & \\
\hline
\end{tabular}

Fuente: Elaboración propia.

Se puede observar que dentro de la matriz de ponderación de los 7 criterios evaluados y una vez realizado el promedio de ponderación por cada criterio de las 18 encuestas realizadas, el criterio que consideran los diversos actores encuestados (INACUCA S.A, Alcaldía de Miranda, comunidad de la vereda El Ortigal y comunidad de la vereda Santa Ana), como de mayor importancia es el criterio 4, que corresponde a la generación y calidad de empleo en actividades relacionadas con el uso de residuos de cosecha. Los criterios más importantes seguidos al criterio 4, son los criterios 1, 2, 3 y 5 , mientras que los criterios que se consideran como de menor importancia dentro de los 7 criterios evaluados por los diversos actores, son los criterios 6 y 7 .

Dentro de la aplicación de la metodología AHP, la consistencia permite asegurar que exista una lógica inherente a la misma de acuerdo a las respuestas obtenidas a través de los diferentes juicios de valor, otorgados por los actores dados los diversos crite- rios relacionados. En este sentido se llevó a cabo la comprobación de la consistencia de los juicios ingresados en la matriz de comparaciones, pues se debe corroborar que al fijar prioridades para elementos o actividades de algún criterio, estos deben ser válidos en la vida real. Pacheco \& Contreras (2008 p.54), mencionan que el AHP "mide la inconsistencia global de los juicios mediante la Proporción de Consistencia, que es el resultado de la relación entre el Índice de Consistencia y el Índice Aleatorio. El Índice de Consistencia es una medida de la desviación de consistencia de la matriz de comparaciones a pares y el Índice Aleatorio es el índice de consistencia de una matriz recíproca aleatoria, con recíprocos forzados, del mismo rango de escala de 1 hasta 9". El valor de la relación de consistencia no debe superar el 10\% para demostrar que es un juicio informado. Para el cálculo de la relación de consistencia se parte de la ecuación:

$$
\lambda \max =V^{*} B
$$


En donde ( $\lambda \max )$ es el máximo valor propio de la matriz de comparaciones a pares; $(\mathrm{V})$ es el vector de prioridades que se obtuvo en la matriz de comparaciones y (B) es la suma de los elementos de cada columna de la matriz a comparaciones a pares $(\mathrm{Pa}-$ checo \& Contreras, 2008).

Para nuestro ejercicio el valor de la relación de consistencia nos dio como resultado 1,70. Sin embargo, a partir de aquí podemos calcular el índice de consistencia (CI) mediante la siguiente ecuación:

$$
C I=(\lambda \max -n) /(n-1)
$$

Donde (n) es el número de criterios que hemos venido trabajando y el valor que nos arroja el índice de consistencia es de $-0,88$. Finalmente para obtener el índice de consistencia nos hace falta el índice aleatorio (RI), el cual se toma de una tabla elaborada por Saaty (1997), que muestra los índices de consistencia para una serie de matrices aleatorias con recíprocos forados, para nuestro caso es de 1,32. Finalmente se resuelve la Relación de Consistencia bajo la ecuación:

$$
(R C=C I / R I)
$$

El resultado fue de $-0,67$, ratificando que los juicios trabajados son consistentes en el ejercicio y que no es necesario reevaluar los juicios nuevamente con los expertos.

El análisis de los indicadores, involucra entonces los valores cualitativos, empleando escalas que permitan su homologación numérica, para lograr esto se definieron cuidadosamente unos atributos con su correspondiente valor numérico y a partir de allí se cuantificaron las entrevistas, los grupos focales y parte de la información secundaria que se obtuvo de literatura relacionada con el proyecto y el área de estudio.

Finalmente el cálculo de índices, consiste simplemente en tomar los valores de los ponderadores previamente hallados y multiplicarlos por los valores normalizados de los indicadores, permite establecer un orden jerárquico dando preferencia aquel que obtuvo mayor puntuación en la evaluación, en este sentido puede observarse que en términos globales es más favorable una situación sin proyecto MDL $(0,37)$ que una situación con proyecto $\operatorname{MDL}(0,47)$, como se evidencia en las Tablas 6 y 7.

\begin{tabular}{|c|c|c|c|c|}
\hline \multirow{2}{*}{ Criterio } & \multirow{2}{*}{ Alternativa } & $\begin{array}{c}\text { Valor } \\
\text { normalizado }\end{array}$ & \multirow{2}{*}{$\begin{array}{c}\text { Ponderador } \\
\text { de cada } \\
\text { criterio }\end{array}$} & \multirow{2}{*}{ Resultado } \\
\hline & & $\begin{array}{l}\text { Promedio de } \\
\text { las fuentes }\end{array}$ & & \\
\hline \multirow{2}{*}{ Criterio 1} & Con & 3.03 & 0.14 & 0.43 \\
\hline & $\operatorname{Sin}$ & 2.99 & 0.14 & 0.43 \\
\hline \multirow{2}{*}{ Criterio 2} & Con & 3.08 & 0.14 & 0.44 \\
\hline & $\operatorname{Sin}$ & 2.92 & 0.14 & 0.42 \\
\hline \multirow{2}{*}{ Criterio 3} & Con & 1.85 & 0.14 & 0.26 \\
\hline & $\operatorname{Sin}$ & 0.95 & 0.14 & 0.14 \\
\hline \multirow{2}{*}{ Criterio 4} & Con & 3.95 & 0.43 & 1.69 \\
\hline & $\operatorname{Sin}$ & 2.53 & 0.43 & 1.08 \\
\hline \multirow{2}{*}{ Criterio 5} & Con & 2.98 & 0.14 & 0.43 \\
\hline & $\operatorname{Sin}$ & 3.54 & 0.14 & 0.51 \\
\hline \multirow{2}{*}{ Criterio 6} & Con & 3.47 & 0.00 & 0.00 \\
\hline & $\operatorname{Sin}$ & 2.14 & 0.00 & 0.00 \\
\hline \multirow{2}{*}{ Criterio 7} & Con & 3.60 & 0.00 & 0.00 \\
\hline & Sin & 2.58 & 0.00 & 0.00 \\
\hline
\end{tabular}

Tabla 6. Cálculo de índices

Fuente: Elaboración propia.

Tabla 7. Establecimiento de ranking global

\begin{tabular}{|c|c|}
\hline SIN PROYECTO & CON PROYECTO \\
\hline 0.37 & 0.47 \\
\hline
\end{tabular}

Fuente: Elaboración propia.

\subsection{Análisis de resultados}

Debido a que el objetivo del estudio se centra en la incidencia que el del proyecto MDL en mención sobre el desarrollo sostenible, fueron analizadas las 
cuatro dimensiones, planteadas en la última cumbre de Río + 20 (ONU, 2012) que culminó en junio de 2012, obteniendo los siguientes resultados:

Se puede observar que dentro de la matriz de ponderación de los 7 criterios evaluados, durante la fase de salida de campo y una vez realizado el promedio de ponderación por cada criterio de las 18 encuestas realizadas:

- El criterio que los diversos actores encuestados (Incauca S.A., Alcaldía de Miranda, comunidad de la vereda El Ortigal y comunidad de la vereda Santa Ana), consideraron de mayor importancia es el número 4, que corresponde a: $\mathrm{La}$ Generación y calidad de empleo en actividades relacionadas con el uso de residuos de cosecha.

- Los criterios que le siguieron en importancia, son los criterios $1,2,3$ y 5 .

- Los criterios que se consideraron como de menor importancia dentro de los 7 criterios evaluados por los diversos actores, fueron los criterios 6 y 7 .

\subsubsection{Dimensión de sostenibilidad ambiental}

La metodología permitió establecer que la percepción frente a los efectos de la gestión ambiental tanto en el agua, el suelo, la biodiversidad y el aire no representan diferencias importantes entre las prácticas llevadas a cabo antes del proyecto y las prácticas llevadas a cabo a partir del proyecto MDL, dicha afirmación se valida porque el primer criterio analizado "Efectos en la gestión ambiental percibidos por el uso de los residuos de cosecha", presenta participaciones del 0,43 ambas situaciones (con y sin proyecto).

Sin embargo, es importante resaltar que a partir del momento en que se da inicio a los procesos propios del MDL, surge un cambio en la combustión que se lleva a cabo al interior de la planta favoreciendo el aire (reduciendo las emisiones de GEI), pero, como dicha combustión parte de los residuos de cosecha cambia la forma en la que dichos residuos son recogidos, involucrando maquinaria tecnificada que no favorece las condiciones del suelo y la biodiversidad, entre algunos otros componentes.

\subsubsection{Dimensión de inclusión social}

Los indicadores relacionados con la dimensión social del desarrollo sostenible, diseñados para revisar la generación de beneficios no monetarios, fueron orientados hacia el número de programas de inclusión social asociados al uso de residuos de cosecha y la efectividad de tales programas, en tal sentido, la metodología demuestra que la situación "con proyecto" favorece más al desarrollo social del municipio con una participación promedio del 0,44 frente a la situación "sin proyecto" con una participación de tan solo 0,42 .

Es importante reconocer la iniciativa planteada por la empresa Incauca S.A. y acogida por la comunidad de Padilla (vereda ubicada a una hora de la planta), la cual consiste en el desarrollo y puesta en marcha de una cooperativa de corte y transporte de hoja de caña (residuos utilizados en el nuevo proceso de sustitución de combustibles). Al mismo tiempo, se hace necesario presentar las inconformidades manifestadas por otras veredas más cercanas a la planta de Incauca S.A. en Miranda como la vereda El Ortigal y Santa Ana quienes manifiestan que tal iniciativa no genera beneficios tangibles para ellos. Debido a la negativa de participar por parte de las veredas cercanas, la empresa de vio obligada a socializar el proyecto con las veredas más alejadas, encontrando aceptación en la vereda Padilla, donde finalmente se llevó a cabo la iniciativa social.

\subsubsection{Dimensión de desarrollo económico}

En la dimensión de Desarrollo Sostenible, se analizó un criterio denominado "Inversión atraída para el aprovechamiento de residuos de cosecha", el cual obtuvo una de las menores participaciones en el desarrollo económico del municipio con tan solo un 0,14 . Cabe mencionar, que la información disponible frente a la inversión atraída por el MDL, no logró ser ratificada en fuentes secundarias de información y tampoco con los grupos focales llevados a cabo a lo largo del trabajo de campo, esta informa- 
ción fue proporcionada únicamente por los dueños y desarrolladores del proyecto en Incauca S.A.

En esta misma dimensión, encontramos los criterios con mayor y menor información y discusión de todos los analizados por la metodología; aquel más importante de todo el análisis es el denominado "generación y calidad del empleo en actividades relacionadas con el uso de residuos de cosecha" y se encontró, que la alternativa sin proyecto tiene una menor participación $(1,08)$ frente a la alternativa con proyecto $(1,69)$. A partir de la información cualitativa se puede inferir que la alternativa sin proyecto requiere emplear más recurso humano en la recolección del bagazo y posteriormente esta labor fue tecnificada y reemplazada por maquinaria que no permite el empleo de la misma cantidad de personas, pese a ello, la alternativa con proyecto logró desarrollar una cooperativa de corte y transporte de hoja de caña que vincula laboralmente a muchos de los empleados ocasionales que existían antes del MDL.

Cabe resaltar que la compañía Incauca S.A. manifiesta que las condiciones laborales para todas las personas vinculadas con la cooperativa de hoja verde mejoraron ostensiblemente, debido a que tienen la oportunidad de formalizar su actividad, cotizar prestaciones sociales e incluso adelantar capacitaciones con la Fundación Carvajal, entidad que apoya a los pobladores y a la empresa en el proceso de puesta en marcha de dicha cooperativa.

Adicionalmente, el criterio denominado "beneficios netos generados por los residuos de cosecha", representa, según la metodología, una participación promedio en el desarrollo social del municipio, con un 0,43 , esto puede ser explicado de acuerdo a algunas fuentes de información secundaria y la entrevista llevada a cabo a la compañía dueña y desarrolladora del proyecto, en donde se manifiesta que gracias al MDL se han adelantado importantes planes sociales que benefician a la comunidad.

\subsubsection{Dimensión de gobernabilidad}

La dimensión de gobernabilidad fue analizada por medio del criterio denominado "fortalecimiento de organizaciones civiles y de procesos participativos para el uso de residuos de cosecha", tal criterio tiene una participación nula bajo los dos escenarios analizados, del 0,00. Esto refleja una clara problemática de interacción de Incauca S.A. con la comunidad de Miranda, dada una debilidad en los procesos de participación por parte de los representantes de la comunidad involucrada en el presente estudio.

Esto podría obedecer a lo que se evidencia en la alternativa sin proyecto (según la percepción cualitativa) cuando el desconocimiento de los procesos participativos implementados en la gestión ambiental del municipio y la poca participación de la comunidad en procesos de planificación y aprovechamiento de los residuos de cosecha corresponde a un elemento de conflicto.

Sin embargo, como se mencionó anteriormente, la Alcaldía de Miranda reconoce la participación de la compañía Incauca S.A. en el adelanto de alternativas que van en favor del ambiente, a pesar de dimensionar algunos contrastes de la visión del desarrollo local entre las comunidades y la empresa, la cual ha aportado en este sentido a través de interesantes iniciativas como el de la cooperativa de Hoja Verde.

\section{Conclusiones}

La presente investigación se llevó a cabo con la metodología de análisis multicriterio, la cual busca integrar las diferentes dimensiones de la realidad de una problemática, con el fin de dar una visión integral y un mejor acercamiento a la realidad. En el ejercicio práctico se pudo observar que esta metodología permite identificar la pluralidad de percepciones de los actores que intervienen en el proceso, así como también sus preferencias, convergencias y divergencias. A pesar de que se reconocen ciertas limitaciones inherentes a la metodología y al mismo trabajo de campo, de acuerdo a la subjetividad relacionada con la selección de los actores, no compromete la validez de los resultados y hallazgos obtenidos, más aún cuando el estudio se ciñó a los presupuestos teóricos que involucra un enfoque tanto cualitativo como cuantitativo de investigación. 
Atendiendo al objetivo de la investigación de analizar los proyectos de Mecanismo de Desarrollo Limpio (MDL) del sector de generación de energía eléctrica por biomasa en Colombia: en particular el proyecto MDL de sustitución de combustibles en Incauca S.A., y tal como fue mencionado en la metodología, se desarrolló el presente trabajo de investigación bajo dos escenarios: el primero contemplando la situación "sin proyecto", es decir, analizando las diferentes situaciones antes del proyecto y en ausencia del mismo; y el segundo contemplando la situación "con proyecto", analizando las situaciones presentadas desde el diseño y puesta en marcha de éste.

De acuerdo a lo anterior, como resultado definitivo se puede evidenciar que no existe una diferencia importante entre la situación "con proyecto" y la situación "sin proyecto", sin embargo al analizar de manera global el ranking de las dos alternativas es evidente que los actores involucrados prefieren la situación sin proyecto a la situación con proyecto, en otras palabras, puede deducirse del presente estudio que la contribución al desarrollo sostenible del Municipio de Miranda, Cauca, no está determinada por la puesta en marcha del proyecto MDL. De esta manera la información proporcionada por las diferentes fuentes de información consultadas y validadas con la metodología de análisis multicriterio indican que las situaciones presentadas con anterioridad al proyecto MDL o en ausencia del mismo resultan contribuir prácticamente en la misma medida con la situación actual en presencia del MDL al desarrollo social del municipio.

Por lo tanto, el método AHP es claro al indicar que los promedios de los datos recuperados son ínfimamente distintos entre estas situaciones, debido a que la diferencia entre estos valores es tan solo del 0,10 puntos, donde la contribución al desarrollo sostenible del Municipio de Miranda (Cauca), está algo más aventajada con la situación en ausencia del proyecto que con el mismo.

Cabe resaltar que debido a que uno de los objetivos del MDL es la propagación de alternativas favorables para el ambiente que reduzcan GEI, el proyecto de Incauca S.A. cumple con tal exigencia, al realizar el cambio en la combustión al interior de la planta favoreciendo el aire dado el otorgamiento de los CER's asociados al proyecto, pero, a pesar de ello se incorpora maquinaria tecnificada que no necesariamente favorece las condiciones del suelo y la biodiversidad y asimismo desplaza la mano de obra local.

Es evidente que si bien los proyectos MDL representan alternativas para incidir en el desarrollo sostenible de los países en vías de desarrollo, no todos tienen el reconocimiento por parte de algunos actores, dentro de ellos las comunidades aledañas a los mismos, en donde se observa el impacto correspondiente. En este sentido es de gran relevancia examinar más de cerca la interacción institucional Empresa -Estado- Comunidad, para optimizar estos procesos, los cuales deberán redundar en resultados de desarrollo sostenible local como un concepto multidimensional.

La mayor preocupación de los actores (Incauca S.A., Alcaldía de Miranda, comunidad de la vereda El Ortigal y comunidad de la vereda Santa Ana) gira en torno a las condiciones de empleo, de tal modo que el criterio 4 (generación y calidad de empleo en actividades relacionadas con el uso de residuos de cosecha), debe responder a las condiciones que la comunidad necesita, pues, la oferta de empleo es poca y la calidad del mismo no garantiza un nivel de vida favorable.

A pesar de que el proyecto MDL fue socializado a la comunidad antes de su implementación y allí se especificaron los beneficios que de este podrían surgir como la creación de la cooperativa de corte y transporte de caña "Hoja Verde", la comunidad de veredas cercanas al ingenio (El Ortigal y Santa Ana) decidieron no hacer parte de la cooperativa, en consecuencia, sólo participó la vereda Padilla (ubicada a una hora de la planta). Posteriormente, cuando la cooperativa se puso en funcionamiento y se brindaron beneficios y privilegios a los socios se negó toda posibilidad de ingreso de las demás veredas circundantes, impidiendo con esto que el proyecto y la iniciativa que surgió del mismo pudiese llegar a tener mayor acogida, es por esto que la empresa Incauca S.A. junto con la fundación Carvajal (apoyo 
en los procesos de diseño y puesta en marcha de la cooperativa) deben propiciar estos espacios y ampliarlos a un mayor número de beneficiarios.

El presente ejercicio de investigación pretende aportar herramientas analíticas para los tomadores de decisiones de políticas ambientales a nivel local, regional y nacional, constituyéndose igualmente en un elemento de discusión académica y científica en aras de fomentar la aplicación de metodologías alternativas que trasciendan del reduccionismo científico para dar respuesta a la complejidad multidimensional del desarrollo sostenible local.

\section{Referencias}

Alcott, B. (2005). Jevons' paradox. Ecological Economics 54 (1): 9-21. Doi:10.1016/j.ecolecon.2005.03.020

Aleluia, J. \& Leitao, J. (2009). International entrepreneurship and technology transfer: the CDM's reality in China. Munich Personal RePEc Archive, № 16150. Online at: http://mpra. ub.uni-muenchen.de/16150/. Diciembre de 2009.

Alvater E. (1998). Obstáculos en la trayectoria del desarrollo. En libro: Los retos de la globalización. Ensayo en homenaje a Theotonio Dos Santos. Segrera, F. (ed.). UNESCO, Caracas, Venezuela. Disponible en: http://bibliotecavirtual.clacso.org. ar/ar/libros/unesco/alvater.rtf

Angulo, N. (2010) Pobreza, medio ambiente y desarrollo sostenible. Nómadas. Revista Crítica de Ciencias Sociales y Jurídicas, 26.

Barba D., Garavito S., López S., Nieves H., Olarte C., Ortega S. \& Santos L. (2010). Segunda comunicación nacional ante la CMNUCC. Capítulo 3. (Online). Recuperado de: http:// www.slideshare.net/inviertaencolombia/sector-tecnologaslimpias-proexport p. 184).

Canoura, C. (2009). América Latina ante los efectos irreversibles de un planeta más caliente. Primer Informe Regional sobre Cambio Climático. Tierramérica Medio Ambiente y Desarrollo.

Casal, J. \& Mateu, E. (2003). Tipos de Muestreo. Rev. Epidem. Med. Prev., 1: 3-7.

Comas, E., Bosch, A., Cuéllar, M. \& Gamboa, G. (2011). Sostenibilidad de la producción porcina en Cataluña (España). Aplicación del análisis multicriterio. Publicado bajo licencia de Redibec en: http://www.redibec.org/IVO/rev18_01. pdf. Iberoamericana de Economía Ecológica Vol. 18: 1-19.

Cotte, A. \& Pardo, C. (2011). Qualitative comparative analysis (QCA): an application for the industry. Quality \& Quantity International Journal of Methodology, 1: 1-8.

De Quinto, J. \& Ferreira, J. (2005). Instrumentos para el desarrollo de los MDL: una oportunidad para Latinoamérica. UNISCI Discussion Papers.

DNP - Departamento Nacional de Planeación (2015). Objetivos de Desarrollo Sostenible (ODS): Agenda de Desarrollo Post-2015 de la Organización de las Naciones Unidas. Disponible en: https://colaboracion.dnp.gov.co/CDT/Prensa/Publicacio-
nes/05\%20Objetivos\%20de\%20Desarrollo\%20Sostenible\%20 para\%20la\%20web.pdf

Doranova, A., Costa, I. \& Duysters, G. (2009). Knowledge base determinants of technology sourcingin clean development mechanism projects. UNU-MERIT, Keizer Karelplein19, Maastricht 6211TC, The Netherlands. Energy Policy, 38: 5550-5559.

Falconí, F. \& Burbano, R. (2004). Instrumentos económicos para la gestión ambiental: decisiones monocriteriales versus decisiones multicriteriales. Revista Iberoamericana de Economía Ecológica, 1: 11-20.

Fernández, G. \& Escribano, M. (2011). La ayuda a la decisión multicriterio: orígenes, evolución y situación actual. Memorias del V Congreso Internacional de Historia de la Estadistica y de la Probabilidad. Disponible en: http://www.ahepe.es/ VICongreso/descargas/Gabriela_Fdz_Barberis.pdf

Funtowicz, S. \& Ravetz, J. (2004). La ciencia post-normal: ciencia con la gente. Madrid: Icaria.

Huang, Y. \& Barker, T. (2010). The Clean Development Mechanism and low carbon development: A panel data analysis, University of Cambridge. Energy Econ., doi:10.1016/j. eneco.2011.08.010

IDEAM, Ministerio del Medio Ambiente \& Departamento Nacional de Planeación. (2002). Lineamientos de política de cambio climático: resumen ejecutivo.

IDEAM, Ministerio de Ambiente, Vivienda y Desarrollo Territorial \& PNUD. (2006). Estrategias de Reducción de Incertidumbre de las Variables Básicas en la Estimación de Gases de Efecto Invernadero (GEI). (Módulos Cambio en el Uso de la Tierra y Silvicultura, Energía - Sector Transporte y Agricultura).

Incauca S.A. (Ingenio del Cauca S.A. - Incauca S.A.) (2011). Informe de sostenibilidad 2010-2011. Disponible en: http:// www.incauca.com/sites/default/files/archivos/Informe $\% 20$ Sostenibilidad\%202010-2011.pdf

Karakosta, C. Doukas, H. \& Psarras, J. (2009). Directing clean development mechanism towards developing countries' sustainable development priorities. National Technical University of Athens, School of Electrical and Computer Engineering, Energy Policy Unit of the Management and Decision Support Systems Laboratory (EPU-NTUA), 9, IroonPolytechniou Str., 157 - 80, Athens, Greece.

Mejía, C. (2005). Mecanismo de Desarrollo Limpio: una debilidad convertida en oportunidad. Pensamiento y Gestión, (18): 138-150.

Olsen, K \& Fenhann, J. (2008). Sustainable development benefits of clean development mechanism projects A new methodology for sustainability assessment based on text analysis of the project design documents submitted for validation. UNEP Ris $\varnothing$ Centre on Energy, Climate and Sustainable Development (URC), Risø National Laboratory, PO Box 49, 4000 Roskilde, Denmark.

ONU (1992). Organización de Naciones Unidas. Convención marco de las Naciones Unidas sobre cambi1o climático. Washington D.C. ONU.

ONU (1998). Organización de Naciones Unidas. Protocolo de Kyoto de la Convención Marco de las Naciones Unidas sobre el cambio climático. Kyoto: ONU.

ONU (2012). Organización de Naciones Unidas. Conferencia de las Naciones Unidas sobre el Desarrollo Sostenible (Rio+20). El Futuro que Queremos. Río de Janeiro (Brasil). ONU. 2012. A/CONF.216/L.1 
ONU (2014). Organización de Naciones Unidas. El camino hacia la dignidad para 2030: Acabar con la pobreza y transformar vidas protegiendo el planeta. Informe de síntesis del secretariado general sobre la agenda de desarrollo sostenible después de 2015. A/69/700

Pacheco, J \& Contreras, E. (2008). Manual metodológico de evaluación multicriterio para programas y proyectos. Serie Manueales de la CEPAL, № 58: 1-111. Instituto Latinoamericano y del Caribe de Planificación Económica y Social (ILPES).

Pierri, N. (2001). Historia del concepto de desarrollo sustentable. Capítulo II: 287-81. En: De Pierri, N. \& Foladori, G. (2001) ¿Sustentabilidad? Desacuerdos sobre el desarrollo sustentable, Uruguay: Trabajo y Capital.

Saaty, T. (1977). A scaling method for priorities in hierarchical structures. Journal of mathematical psychology 15(3): 234-281.

Saaty, T. (1987). The Analytic Hierarchy Process-What It Is And How It Is Used. Mat/d Modelling, 9 (3-5): 161-176.

Saaty, T. (1997). Toma de Decisiones Para Líderes. Universidad de Santiago de Chile.

Saaty, T. (2003). Decision-making with the AHP: Why is the Principal Eigenvector necessary? European Journal of Operational Research, 145(1): 85-91.
Sabogal J., Hurtado E. \& Moreno E. (2010). Evaluación de los proyectos de mecanismo de desarrollo limpio presentados a la UNFCCC: los criterios de sostenibilidad entre 2004 y 2008. Revista Facultad de Ciencias Económicas, 18 (1).

Sandoval, C. (2002). Investigación Cualitativa. Programa de Especialización en Teoría Métodos y Técnicas de Investigación Social. Instituto Colombiano para el Fomento de la Educación Superior ICFES

SDSN - Red de Soluciones para el Desarrollo Sostenible. (2013) Una Agenda de Acción para el Desarrollo Sostenible. Disponible en: www.unsdsn.org.

UNEP (2014). Programa de las Naciones Unidas para el Medio ambiente. Centre on Energy Climate and Sustainable Developent. Copenhagen (Dinamarca). Recuperado de http:// http://www.cdmpipeline.org/

Vergara, C. \& Lozada, J. (2015). Método de proceso de jerarquía analítica: potencialidades y limitaciones en Colombia: caso de estudio proyecto MDL Incauca S.A. Memorias IX Congreso de Investigaciones Universidad Militar Nueva Granada. Bogotá.

Villavicencio, A. (2004). Mitos y realidad del mecanismo de desarrollo limpio. Revista Iberoamericana de Economía Ecológica, 1: 56-65. 